\title{
Tibial Osteomyelitis Caused by Carbapenem-Resistant Enterobacter cloacae
}

\author{
David J. Cinats $^{a} \quad$ Evgeny Strovski $^{b} \quad$ Cheng-Han Lee $^{c} \quad$ Darius Viskontas $^{d}$ \\ aDepartment of Orthopaedic Surgery, SUNY Upstate Medical University, \\ Syracuse, NY, USA; ${ }^{b}$ Department of Radiology, Surrey Memorial Hospital, University of \\ British Columbia, Surrey, BC, Canada; 'Department of Pathology and Laboratory Medicine, \\ BC Cancer, Surrey Memorial Hospital, Surrey, BC, Canada; dDepartment of Orthopaedics, \\ Royal Columbian Hospital, University of British Columbia, Vancouver, BC, Canada
}

\section{Keywords}

Carbapenemase-producing organism $\cdot$ New Delhi metallo- $\beta$-lactamase $\cdot$ Osteomyelitis $\cdot$ Tibia fracture - Ewing sarcoma

\begin{abstract}
A 31-year-old man presented with a symptomatic tibial non-union following an open tibia fracture sustained in India. The open fracture was persistently draining for 8 months following the initial injury. The decision was made to revise his tibia fracture to promote union. Intraoperative cultures of the non-union site during the revision procedure revealed osteomyelitis caused by carbapenemase-producing Enterobacter cloacae which was multi-drug resistant to all antibiotics except for tigecycline. The patient was treated successfully with a tigecyclineimpregnated antibiotic spacer and intravenous tigecycline with repeat bone cultures demonstrating eradication of the infection. Eight weeks into treatment (1 year from initial injury), the patient developed new pain and swelling in the adjacent proximal fibula of the limb treated
\end{abstract}


for osteomyelitis. MRI and biopsy revealed Ewing sarcoma. The patient underwent transfemoral amputation. This report describes a management protocol of carbapenemase-resistant post-traumatic osteomyelitis with no evidence of infection recurrence.

(C) 2020 The Author(s)

Published by S. Karger AG, Basel

\section{Introduction}

Tibial osteomyelitis after open tibia fractures is a well-known complication with incidences from 49.4 to $63.2 \%$ [1]. Infections caused by antibiotic-resistant bacteria are becoming increasingly problematic. Carbapenemase-producing organisms (CPOs) are a relatively new group of bacteria that are resistant to a wide spectrum of antibiotics, including the carbapenems, which are often seen as the "last line" for treating infections caused by these bacteria [2]. We present a case of tibial osteomyelitis caused by carbapenemase-producing Enterobacter cloacae that was treated with systemic tigecycline resulting in sterile intra-operative cultures and resolution of symptoms. CPO-mediated tibial osteomyelitis has not been previously reported to our knowledge.

\section{Case Report}

A 31-year-old male sustained an open tibial shaft fracture in India after a motorcycle collision. The patient underwent irrigation and debridement of the open wound with intramedullary stabilization. The patient developed purulent drainage from the skin grafting site that was treated with serial bedside debridement (Fig. 1). The patient returned to Canada for orthopaedic follow-up approximately 8 months after the injury. Radiographs demonstrated a lack of union at the fracture site (Fig. 2) with persistent pain. A work-up for infection demonstrated an elevated C-reactive protein of $44 \mathrm{mg} / \mathrm{L}$. A septic non-union was suspected and the patient was taken to the operating room for removal of the nail, debridement and culture of the non-union, and antibiotic-impregnated polymethylmethacrylate (PMMA)-coated intramedullary nail and spacer insertion in order to treat the presumed osteomyelitis with a future induced membrane technique to address the bone loss. Vancomycin $2 \mathrm{~g}$ and tobramycin $3.6 \mathrm{~g}$ were used in the PMMA spacer and nail. Three of five bone cultures taken intra-operatively grew E. cloacae resistant to multiple antibiotics (including the antibiotics used in the construct) with the only exceptions being tigecycline, aztreonam, and colistin (Table 1). The minimum inhibitory concentration of tigecycline was $1 \mathrm{mg} / \mathrm{L}$. The patient was treated with intravenous tigecycline $50 \mathrm{mg}$ every $12 \mathrm{~h}$ for 4 weeks. Since the initial irrigation and debridement with antibiotic spacer/nail insertion was performed with antibiotics to which the E. cloacae was resistant, the decision was made to perform a revision procedure using PMMA containing tigecycline. Four weeks after the initial antibiotic-coated nail insertion, the previous nail and spacer were removed and a new antibiotic-coated nail and spacer containing tigecycline was inserted. Five intraoperative bone samples were taken during this revision surgery to assess the response to antibiotics, and after 14 days of culture there was no growth in any of the samples, indicating successful eradication of the infection. 


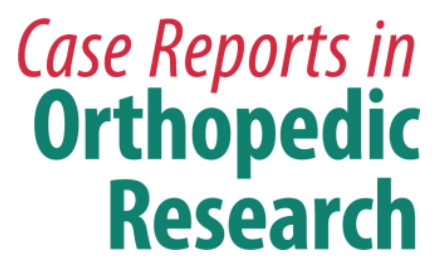

Case Rep Orthop Res 2020;3:16-24

DOI: $10.1159 / 000506414$

(c) 2020 The Author(s). Published by S. Karger AG, Basel www.karger.com/cio

Cinats et al.: Tibial Osteomyelitis Caused by Carbapenem-Resistant E. cloacae

During a routine post-operative visit 4 weeks after the second PMMA spacer insertion, the patient complained of a swelling around the proximal fibula and a foot drop. Radiographs revealed a permeative, "moth-eaten" appearance of the proximal fibula (Fig. 3). A gadoliniumenhanced MRI suggested a primary soft tissue or bone sarcoma (Fig. 4). The tumour was positive for CD99 (Fig. 5) and ERG, and negative for myogenin, desmin, keratin, synaptophysin, chromogranin, S100, and CD45 by immunohistochemistry. Fluorescence in situ hybridization analysis demonstrated the presence of EWSR1 and FLI1 genetic rearrangement, which confirmed the diagnosis of Ewing sarcoma. The patient underwent transfemoral amputation. At the final 12-month follow-up, the patient is doing well with no evidence of recurrence of New Delhi metallo- $\beta$-lactamase (NDM)-mediated infection or Ewing sarcoma.

\section{Discussion}

Tibial osteomyelitis and non-union are devastating complications after open tibial fractures with an incidence of 5-50\% [1]. Over the past decade, there has been a worldwide emergence of bacteria that have acquired resistance to a wide spectrum of $\beta$-lactam antibiotics, rendering these antibiotics ineffective [2]. The widest range of $\beta$-lactam-resistant bacteria are the CPOs. Although fears around multi-drug-resistant gram-positive bacteria such as methicillin-resistant Staphylococcus aureus (MRSA) have been widely reported, clinical microbiologists are in increasing agreement that these CPOs pose the greatest risk to public health [3]. CPO-mediated infections have high rates of mortality of up to $50 \%$ and there are few effective antibiotics available for treatment [4].

The first case of an NDM-mediated infection occurred in 2008. NDM is now the most common carbapenemase in the Indian subcontinent with a few cases reported in North America. Osteomyelitis caused by CPO has been rarely reported in the literature. The first case of CPOmediated osteomyelitis caused by E. cloacae was reported in 2019 [5]. This multi-drug-resistant infection was successfully treated with a hexapod frame, systemic colistin, sulbactam, and fosfomycin as well as local delivery of colistin-impregnated methanesulphonate [5]. Treatment of CPO-mediated infections remains difficult as few antibiotics exist that are effective in eradication. Most CPO infections are treated with colistin, tigecycline, and fosfomycin [2]. Despite resistance to carbapenems, some antibiotic regimens using dual carbapenem therapy may be effective due to synergistic effects [6]. Combining these various antimicrobials is more effective at reducing mortality rates than monotherapy alone [2]. Tigecycline is a minocycline-derived antibiotic which was originally approved for use in skin and intra-abdominal infections. It is parentally administered and demonstrates good activity against both gram-positive and gram-negative bacteria. Tigecycline has been previously used to treat bone and joint infections in multi-drug-resistant gram-positive bacteria [7, 8]. Intravenously administered tigecycline demonstrates good penetration into bone with high residual levels of the antibiotic in bone tissue despite being cleared rapidly in serum once the administration of the antibiotic has completed $[9,10]$. Tigecycline exists as a powdered preparation and, when combined with PMMA, is heat stable and demonstrates clinically significant antibiotic elution that lasts up to a week [11]. In this case, the tissue and bone samples taken during the second surgery (after 4 weeks of intravenously administered tigecycline in isolation) did not grow 
any bacteria. This suggests that intravenous tigecycline without the need for tigecycline-impregnated cement spacers may be sufficient for eradication of infected tissue. However, it should be noted that true eradication of infection, as demonstrated by bony union, was not observed as the limb was amputated due to the Ewing sarcoma prior to signs of healing.

This is an atypical presentation for Ewing sarcoma in that the patient developed the sarcoma immediately adjacent to a soft tissue and bone infection. Ewing sarcoma is caused by a balanced translocation resulting in a fusion protein that acts as an oncogenic transcription factor [12]. Ewing sarcoma is thought not to occur as a secondary neoplasm, as the only predisposing factors are tumour syndromes. Ewing sarcoma has been known to mimic osteomyelitis, with a number of cases reported in the literature [13-17]. Interestingly, there is one other case published where a 24-year-old patient with suspected osteomyelitis subsequently developed Ewing sarcoma [18]. The patient was treated for recurring humeral shaft osteomyelitis and developed Ewing sarcoma in the humerus 3 years after the initial diagnosis of osteomyelitis [18]. As there is no association between osteomyelitis and Ewing sarcoma, the cooccurrences of these entities is likely coincidental, although unusual nonetheless.

In conclusion, health care providers should be aware of the threat of CPO-mediated orthopaedic infections and the various treatment options available for treatment. This case report highlights a treatment regimen for NDM-mediated tibial osteomyelitis using systemic tigecycline, with no evidence of infection recurrence after 6 weeks of treatment. This case is unique in the development of a Ewing sarcoma approximately 1 year after the initial injury and 8 weeks into treatment of the osteomyelitis. As the incidence of these infections increases, an understanding of effective antimicrobial and surgical therapy is essential for eradication of infection.

\section{Statement of Ethics}

The subject in this case report provided consent for publication of this case as well as use of clinical images and radiographic imaging.

\section{Disclosure Statement}

The authors have no conflicts of interest to declare.

\section{Funding Sources}

The authors have no funding sources to report. 


\section{Author Contributions}

All of the authors listed on this publication contributed substantially to the conceptualization of the manuscript, data collection and analysis, and writing or proofreading the manuscript.

\section{References}

1 Khatod M, Botte MJ, Hoyt DB, Meyer RS, Smith JM, Akeson WH. Outcomes in open tibia fractures: relationship between delay in treatment and infection. J Trauma. 2003 Nov;55(5):949-54.

2 Bonomo RA, Burd EM, Conly J, Limbago BM, Poirel L, Segre JA, et al. Carbapenemase-producing organisms: a global scourge. Clin Infect Dis. 2018 Apr;66(8):1290-7.

3 Kumarasamy KK, Toleman MA, Walsh TR, Bagaria J, Butt F, Balakrishnan R, et al. Emergence of a new antibiotic resistance mechanism in India, Pakistan, and the UK: a molecular, biological, and epidemiological study. Lancet Infect Dis. 2010 Sep;10(9):597-602.

4 Martin A, Fahrbach K, Zhao Q Lodise T. Association between carbapenem resistance and mortality among adult, hospitalized patients with serious infections due to Enterobacteriaceae: results of a systematic literature review and meta-analysis. Open Forum Infect Dis. 2018 Jun;5(7):ofy150.

5 Siebenbürger G, Grabein B, Schenck T, Kammerlander C, Böcker W, Zeckey C. Eradication of Acinetobacter baumannii/Enterobacter cloacae complex in an open proximal tibial fracture and closed drop foot correction with a multidisciplinary approach using the Taylor Spatial Frame®: a case report. Eur J Med Res. 2019 Jan;24(1):2.

6 Poirel L, Kieffer N, Nordmann P. In vitro evaluation of dual carbapenem combinations against carbapenemase-producing Enterobacteriaceae. J Antimicrob Chemother. 2016 Jan;71(1):156-61.

7 Asseray N, Bemer P, Corvec S, Boutoille D, Touchais S, Navas D. Tigecycline option for the treatment of bone and joint infections caused by multidrug-resistant Staphylococcus epidermidis. Joint Bone Spine. 2012 Jan;79(1):97-9.

8 Thompson S, Townsend R. Pharmacological agents for soft tissue and bone infected with MRSA: which agent and for how long? Injury. 2011 Dec;42 Suppl 5:S7-10.

9 Bhattacharya I, Gotfried MH, Ji AJ, Saunders JP, Gourley I, Diehl A, et al. Reassessment of tigecycline bone concentrations in volunteers undergoing elective orthopedic procedures. J Clin Pharmacol. 2014 Jan;54(1):70-4.

10 Landersdorfer CB, Bulitta JB, Kinzig M, Holzgrabe U, Sörgel F. Penetration of antibacterials into bone: pharmacokinetic, pharmacodynamic and bioanalytical considerations. Clin Pharmacokinet. 2009;48(2):89124.

11 Nichol T, Smith TJ, Townsend R, Stockley I, Akid R. Analysis of linezolid and tigecycline as candidates for local prophylaxis via antibiotic-loaded bone cement. J Antimicrob Chemother. 2017 Feb;72(2):410-6.

12 Choi EY, Gardner JM, Lucas DR, McHugh JB, Patel RM. Ewing sarcoma. Semin Diagn Pathol. 2014 Jan;31(1):39-47.

13 Brazão-Silva MT, Fernandes AV, Faria PR, Cardoso SV, Loyola AM. Ewing's sarcoma of the mandible in a young child. Braz Dent J. 2010 Jan;21(1):74-9.

14 Durbin M, Randall RL, James M, Sudilovsky D, Zoger S. Ewing's sarcoma masquerading as osteomyelitis. Clin Orthop Relat Res. 1998 Dec;357:176-85.

15 Henninger B, Glodny B, Rudisch A, Trieb T, Loizides A, Putzer D, et al. Ewing sarcoma versus osteomyelitis: differential diagnosis with magnetic resonance imaging. Skeletal Radiol. 2013 Aug;42(8):1097-104.

16 Lyall HA, Constant CR, Wraight EP. Case report: Ewing's sarcoma in distal tibial metaphysis mimicking osteomyelitis. Clin Radiol. 1993;48(2):140-2.

17 Jordanov MI, Block JJ, Gonzalez AL, Green NE. Transarticular spread of Ewing sarcoma mimicking septic arthritis. Pediatr Radiol. 2009;39(4):381-4.

18 Tow BP, Tan MH. Delayed diagnosis of Ewing's sarcoma of the right humerus initially treated as chronic osteomyelitis: a case report. J Orthop Surg (Hong Kong). 2005;13(1):88-92. 


\section{Case Reports in Orthopedic Research}

www.karger.com/cio

Cinats et al:: Tibial Osteomyelitis Caused by Carbapenem-Resistant $E$. cloacae

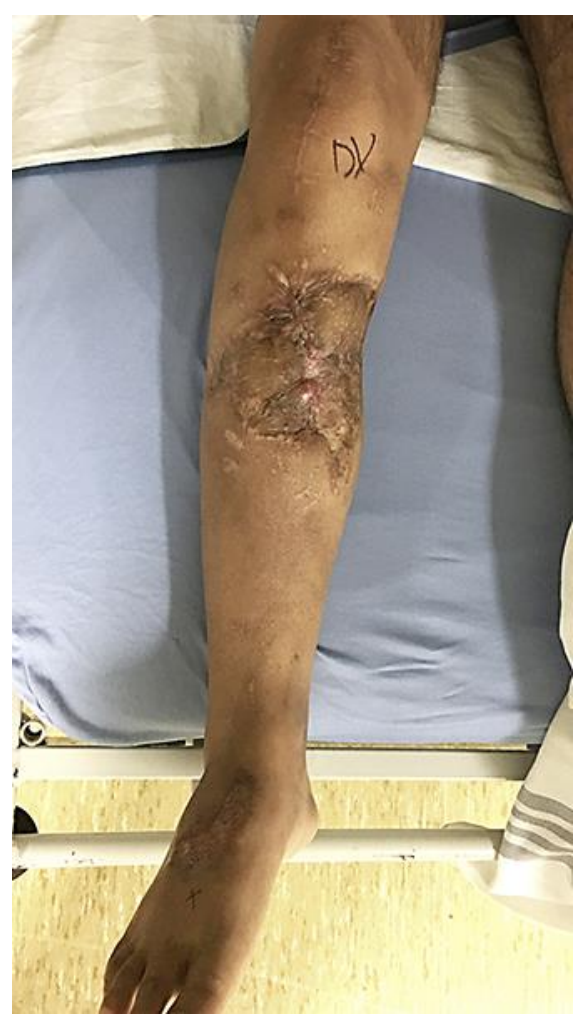

Fig. 1. Clinical photograph of the lower extremity after the initial intramedullary nail insertion and soleus flap with skin grafting. The open injury and soft tissue loss occurred on the proximal anteromedial aspect of the tibia. 


\section{Case Reports in Orthopedic Research}

Case Rep Orthop Res 2020;3:16-24 DOI: $10.1159 / 000506414$ (c) 2020 The Author(s). Published by S. Karger AG, Basel Cinats et al.: Tibial Osteomyelitis Caused by Carbapenem-Resistant E. cloacae

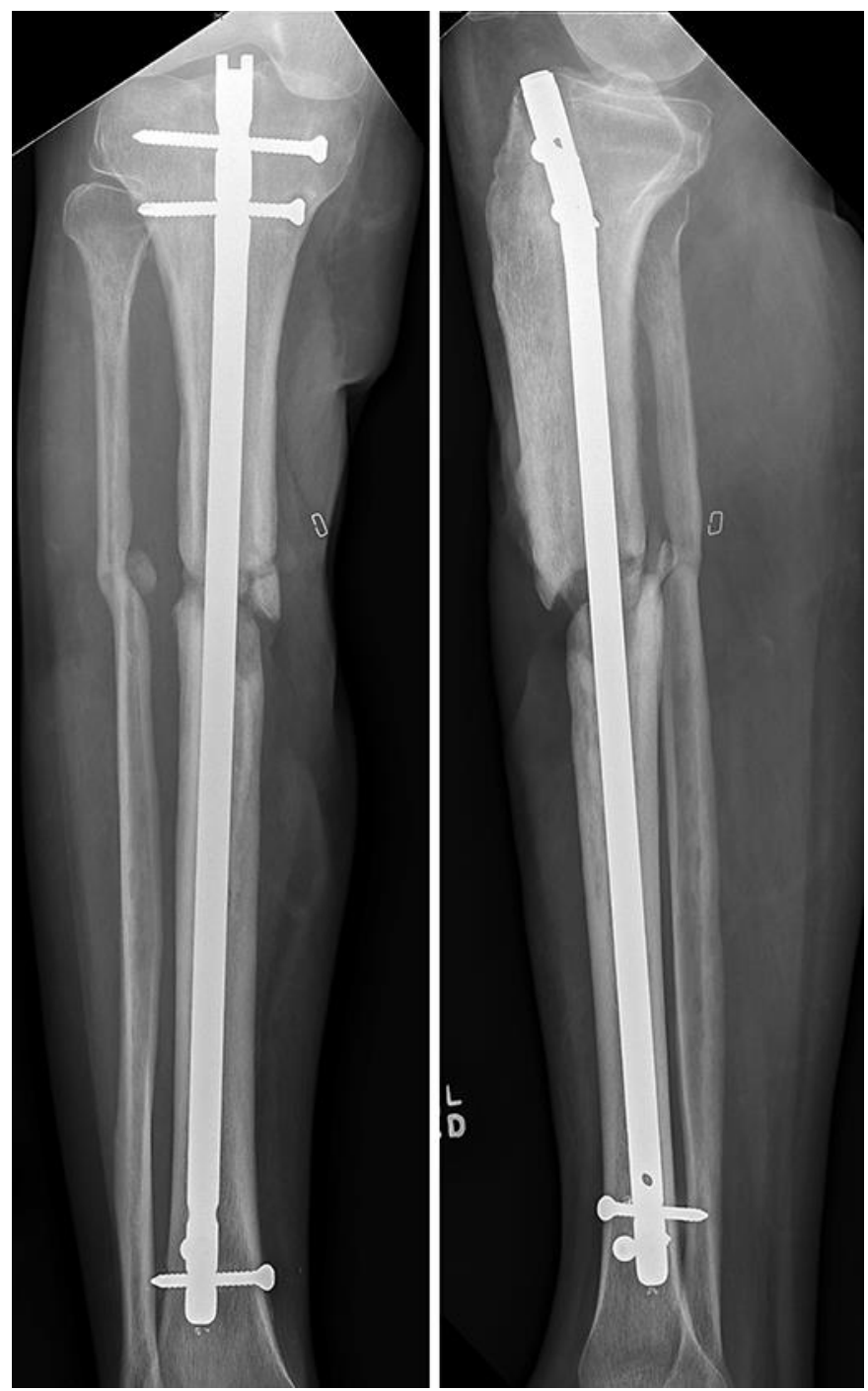

Fig. 2. Anteroposterior and lateral radiographs of the tibia demonstrating a non-union at the fracture site. The tibial nail was intact. 


\section{Case Reports in Orthopedic Research}

Case Rep Orthop Res 2020;3:16-24

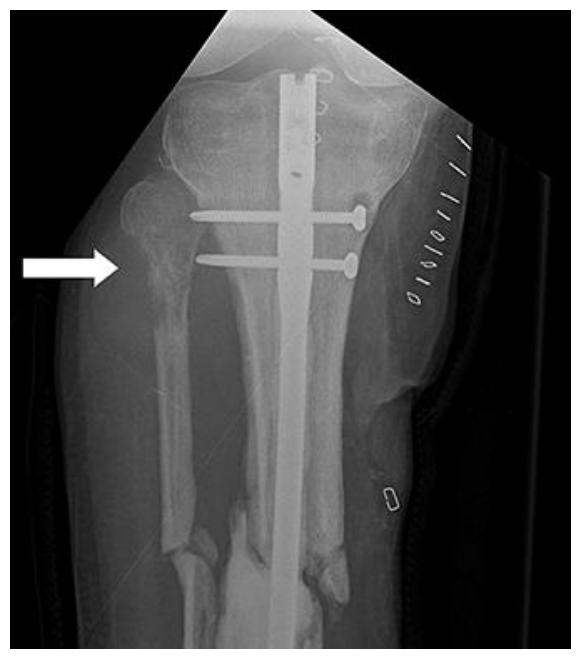

Fig. 3. Anteroposterior radiograph of the proximal tibia taken 6 weeks after the first revision surgery. The patient presented with a new pain and swelling to the lateral aspect of the knee. The proximal fibula demonstrates a "moth-eaten" appearance consistent with a sarcoma (indicated by white arrow).
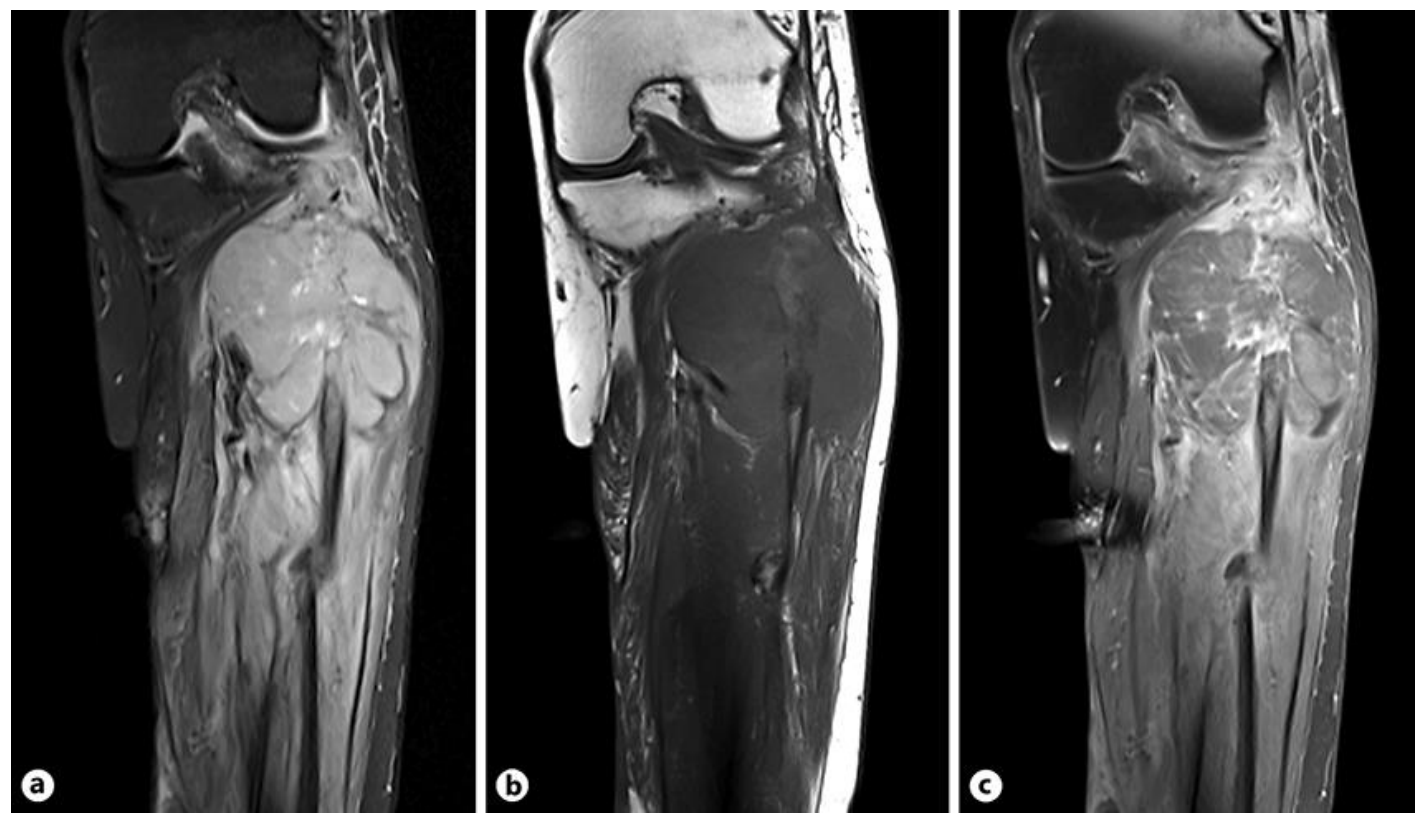

Fig. 4. Representative T1 (a) and T2 (b) and post-gadolinium (c) coronal MRI of the tibia demonstrating a heterogeneous, enhancing mass originating in the proximal fibula which has invaded the anterolateral compartment of the lower extremity consistent with a primary sarcoma of bone. 


\section{Case Reports in Orthopedic Research}
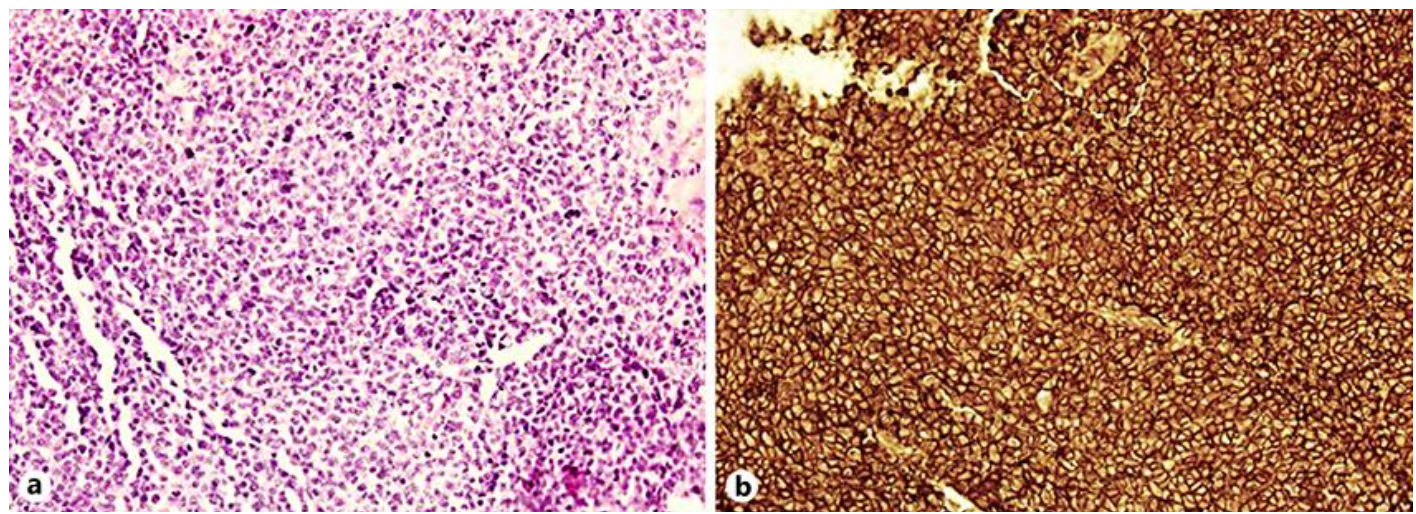

Fig. 5. Pathology of the core needle biopsy showing a cellular malignant small round blue cell tumour with no matrix production (a) and strong diffuse membranous CD99 immunopositivity (b).

Table 1. Resistance profile of Enterobacter cloacae

\begin{tabular}{ll}
\hline Antibiotic & E. cloacae sensitivity \\
\hline Meropenem & $\mathrm{R}$ \\
Imipenem & $\mathrm{R}$ \\
Cefazolin & $\mathrm{R}$ \\
Ampicillin & $\mathrm{R}$ \\
Amikacin & $\mathrm{R}$ \\
Tobramycin & $\mathrm{R}$ \\
Gentamycin & $\mathrm{R}$ \\
Ciprofloxacin & $\mathrm{R}$ \\
Septra & $\mathrm{R}$ \\
Tigecycline & $\mathrm{S}$ \\
Aztreonam & $\mathrm{S}$ \\
Colistin & $\mathrm{S}$ \\
\hline
\end{tabular}

Antibiotic resistance profile of the carbapenemase-producing $E$. cloacae isolated from the intra-operative cultures of tibial bone taken during the first revision procedure to treat the suspected infected nonunion of the tibia. $\mathrm{R}$, resistant; $\mathrm{S}$, sensitive. 